\title{
Optoelectronic Properties of F-co-doped PTO Thin Films Deposited by Spray Pyrolysis
}

\author{
Benjamin Victor Odari ${ }^{1, *}$, Robinson Juma Musembi ${ }^{2}$, Maxwell Joel Mage to ${ }^{1}$, He rick Othie no ${ }^{3}$, \\ Francis Gaitho ${ }^{1}$, Mwamburi Mghendi ${ }^{4}$, Valentine Muramba ${ }^{1}$ \\ ${ }^{1}$ Department of Phy sics, Masinde Muliro University of Science and Technology, P.O Box 190, 50100, Kakamega, Kenya \\ ${ }^{2}$ Department of Phy sics, University of Nairobi, P.O Box 30197-00100, Nairobi, Kenya \\ ${ }^{3}$ Department of Phy sics, Maseno University, P.O Box Private Bag, Maseno, Kenya \\ ${ }^{4}$ Department of Phy sics, University of Eldoret, P.O Box 1125, Eldoret, Kenya
}

\begin{abstract}
F-co-doped Palladium Tin Oxide (PTO) thin films were pyrolytically deposited on glass substrate at $450^{\circ} \mathrm{C}$ using an alcoholic precursor solution consisting of Tin (IV) Chloride $\left(\mathrm{SnCl}_{4} .5 \mathrm{H}_{2} \mathrm{O}\right)$, Palladium $\mathrm{Ch}$ loride $\left(\mathrm{PdCl}_{2}\right)$ and Ammonium Fluoride $\left(\mathrm{NH}_{4} \mathrm{~F}\right)$. A resistivity of $0.3-6.9 \times 10^{-2} \Omega \mathrm{cm}$ was obtained in F-co-doped PTO films prepared with a Pd content of $3.68 \mathrm{at} \%$ and $\mathrm{F}$ content of $0-23.96 \mathrm{at} \%$ under optimized conditions. The optical properties were studied in the UV/VIS/NIR region. The optical bandgap of the films laid in the range 3.945-4.014 eV. Using dispersion analysis with Drude and Kim terms, optical constants were determined from spectro-photometric measurements for films on glass.
\end{abstract}

Keywords Spray Pyrolysis, Tin Oxide, Codoping, Fluorine and Palladium Doping, Electrical, Optical

\section{Introduction}

Transparent conducting oxide (TCO) films produced using binary co mpounds such as $\mathrm{SnO}_{2}, \mathrm{In}_{2} \mathrm{O}_{3}$ and $\mathrm{ZnO}$ doped with an impurity are in practical use[1]. Doped $\mathrm{SnO}_{2}$ is used in a wide range of applications which include the field of sensors, opacities, transparent electrodes in solar panels and other electrochromic devices, thin film magnetic recording media and as material for Li-ion batteries[2, 3]. However, these developments require improvements in electrical, optical and chemical properties of $\mathrm{SnO}_{2}$ thin films used. New transparent conducting oxide films using ternary compounds such as $\mathrm{Zn}_{2} \mathrm{SnO}_{4}, \mathrm{ZnSnO}_{3}, \mathrm{MgIn}_{2} \mathrm{O}_{4}, \mathrm{Zn}_{2} \mathrm{In}_{2} \mathrm{O}_{5}$ and $\mathrm{In}_{4} \mathrm{Sn}_{3} \mathrm{O}_{12}$ have been reported[1]. In addition to the use of ternary compounds, multicomponent oxides such as $\mathrm{ZnO}-\mathrm{In}_{2} \mathrm{O}_{3}$, $\mathrm{In}_{2} \mathrm{O}_{3}-\mathrm{SnO}_{2}, \mathrm{Zn}_{2} \mathrm{In}_{2} \mathrm{O}_{5}-\mathrm{In}_{4} \mathrm{Sn}_{3} \mathrm{O}_{12}$ and $\mathrm{MgIn}_{2} \mathrm{O}_{4}-\mathrm{Zn}_{2} \mathrm{In}_{2} \mathrm{O}_{5}$ were proposed[1]. The use of ternary and multicomponent oxides whose physical and chemical properties can be controlled by changing their chemical co mpositions, may be used to improve the properties of $\mathrm{SnO}_{2}$ and its applications.

$\mathrm{SnO}_{2}$ film is highly transparent in the visible region, chemically inert, mechanically hard and can resist high temperatures $[4,5,6]$ as it is only attacked by hot concentrated alkalis [3]. It belongs to a class of materials that combines high electrical conductivity with optical transparency and therefore constitutes an important

* Corresponding author:

odarivyc@yahoo.com (Benjamin Victor Odari)

Published online at http://journal.sapub.org/materials

Copyright (C) 2013 Scientific \& Academic Publishing. All Rights Reserved component for the optoelectronic applications[2]. The efficiency in these applications is usually improved by suitably doping the tin oxide for example, doping with $\mathrm{Sb}$ and $\mathrm{F}$ increases the conductivity of tin oxide $[3,7,8,9]$. T in oxide is a crystalline solid with a tetragonal crystal lattice. It is a wide gap, non-stoichiometric semiconductor and behaves as a degenerate n-type semiconductor with a low resistivity $\left(\approx 10^{-3} \Omega \mathrm{cm}\right.$ )[9]. It can exist in two structures belonging to an indirect band gap of about $2.6 \mathrm{eV}[10]$ and direct band gap that ranges from $3.6 \mathrm{eV}$ to $4.6 \mathrm{eV}$ at room temperature $[9,11]$. Studies have shown that $\mathrm{F}$ doped $\mathrm{SnO}_{2}$ films have higher electrical conductivity, optical transmission and infrared reflection than the tin oxide films prepared with other dopants [7] while Pd doped $\mathrm{SnO}_{2}$ films have high sensitivity to reducing gases when used as metal gas sensors[12].

Some of the most popular methods ofdepositing Tin oxide thin films include the following: Plasma enhanced Chemical Vapour Deposition[2], Electron beam Evaporation[6], Reactive sputtering[13], Sol-ge1[14], Electronspun[15], Inkjet printing [16] and Spray pyrolysis $[4,5,7,12]$. Of these methods the spray pyrolysis method represents the less expensive method since it can produce large area, high-quality and low cost thin films[7]. It is also suitable for substrates with complex geometry and can be used for a variety of oxide materials[17]. A previous study[30] has shown that the co-doping of $\mathrm{SnO}_{2}$ based ceramics with $\mathrm{Sb}^{5+}$ and $\mathrm{Zn}^{2+}$, noticeably enhances the properties of $\mathrm{SnO}_{2}$ by combining the advantages of $\mathrm{Sb}^{5+}$ which increases the electrical conductivity and $\mathrm{Zn}^{2+}$ which greatly improves the ceramic density. 
In the present study, we report on the deposition and the characterization of single and co-doped $\mathrm{SnO}_{2}$ thin films prepared by spray pyrolysis technique for optoelectronic applications. The aim of this work is to give an insight on how fixed deposition conditions influence the optical and electrical properties of the produced films with a thickness of $132.5 \pm 10 \mathrm{~nm}$. The optical characteristics (transmittance and reflectance) have been evaluated in the UV-Vis-NIR spectral range. In addition, calculations have been carried out in order to determine the carrier densities as well as the optical mobilities by using the Drude model.

\section{Experimental}

\subsection{Sample Preparation}

The substrates used were ordinary float glass slides which were $1.2 \mathrm{~mm}$ thick and measuring 2.5 by $7.6 \mathrm{~cm}^{2}$. The cleaning procedure involved rubbing the glass slides gently on both sides using a cotton swab soaked in foam made from a mixture of deionized water, liquid detergent and sodium hydroxide in the ratio of $3: 2: 1$. They were then drag wiped using a lens cleaning tissue held at an angle of 45 degrees before being wiped with Isopropyl alcohol and acetone respectively. Lastly, the substrates were ultrasonically cleaned in distilled water for 30 minutes before drying with spray of pressurized air. Spray pyrolys is technique was used to deposit the films at a substrate temperature of $450 \pm 10^{\circ} \mathrm{C}$ using compressed air at 1 bar as the atomization gas. The experimental set up of the in-house-made spray pyrolysis system is as shown in Figure 1. It consisted of a fume chamber, hot plate, spray nozzle of diameter $\sim 1 \mathrm{~mm}$, input gas valve, gas compressor, gas flow meter, conduit tube, thermocouple and pressure gauge. $\mathrm{SnO}_{2}$ films were produced from a precursor solution consisting of Tin (iv) chloride $(99 \%)$ prepared by dissolving completely $6 \mathrm{~g}$ of stannic chloride in $100 \mathrm{ml}$ of ethanol $(99.9 \%)[3,5,6]$.

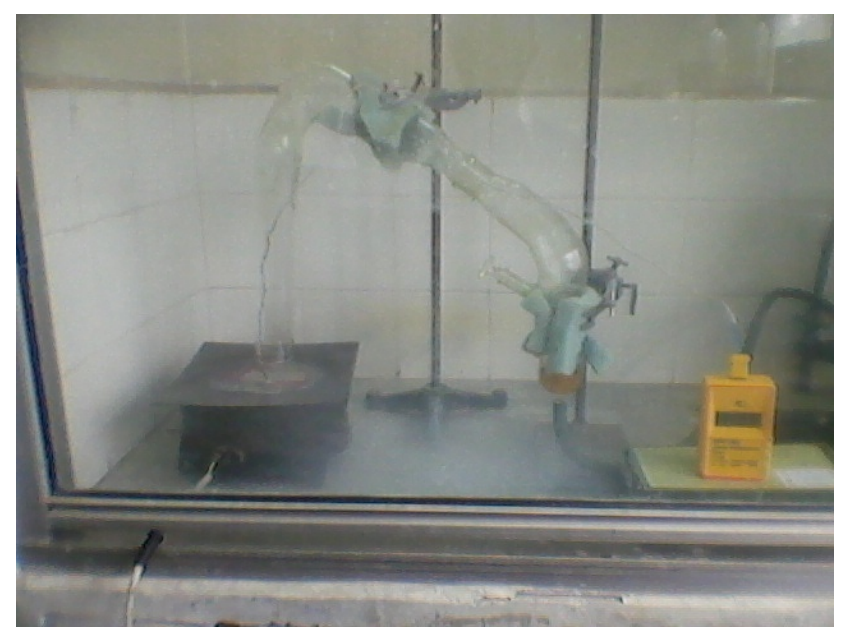

Figure 1. Spray pyrolysis setup

The PTO films were prepared by dissolving $\mathrm{PdCl}_{2}$
$(59-60 \% \mathrm{Pd})$ in ethanol $(99.9 \%)$ then added to the spraying solution at a constant volume. For F:PTO films, varying volumes of a solution of $0.5 \mathrm{~g} \mathrm{NH}_{4} \mathrm{~F}(99 \%)$ in distilled water was added to the starting solution[16,18].

The spraying was done under the following conditions:

- Substrate temperature: $450 \pm 10^{\circ} \mathrm{C}$

- Carrier-gas: Compressed air at 1 bar

- Solution flow rate: $6 \mathrm{ml} / \mathrm{min}$

- Nozzle-to-substrate distance: $33 \mathrm{~cm}$ horizontally $32+3 \mathrm{c}$ m vert ically

After spraying, the films were left to cool with the hot plate before removal for transmittance and reflectance measurements.

\subsection{Sample Characterization}

Spectral normal transmittance $T(\lambda)$ and near normal reflectance $R(\lambda)$ were measured in the wavelength range 300 $<\lambda<2500 \mathrm{~nm}$, on a computerized double beam solid-spec 3700DUV Shimadzu Spectrophotometer equipped with 198851 Barium Sulphate $\left(\mathrm{BaSO}_{4}\right)$ integrating sphere. Barium Sulphate plate was used as a reference for the calculation of optical properties such as band gap, absorption coefficient and refractive index. The thickness of the as-deposited samples of F:PTO were estimated by fitting the experimental spectral data to theoretical spectral data based on Drude and Kim analysis using the SCOUT software[19] in the wavelength range $300 \mathrm{~nm}-2500 \mathrm{~nm}$. Electrical resistivity of the films was calculated from two adjustable parameters of Drude: plasma frequency $\Omega_{\mathrm{p}}$ and damping constant $\gamma$.

\section{Results and Discussion}

\subsection{Analysis of Optical Properties}

In order to compare the transparency of PTO thin films with various F-doping levels, the optical spectra in the UV-VIS-NIR region and near infrared region of the samples were measured. The optical transparency of PTO films and F:PTO films for both experimental and computed spectral are shown in Figure 2.

The films exhibited high transparency in the visible region with an average transmittance of $83 \%$. Maximum transmittance of $85 \%$ for $19.11 \mathrm{at} \% \mathrm{~F}: \mathrm{PTO}$ at wavelength of $540 \mathrm{~nm}$ was recorded. The two weird peaks at about $900 \mathrm{~nm}$ and $1600 \mathrm{~nm}$ occur due to change of detectors since the SolidSpec-3700DUV spectrophotometer used in this study, is equipped with 3 detectors: a photomultip lier tube detector for the ultraviolet and visible regions, and $\mathrm{InGaAs}$ and $\mathrm{PbS}$ detectors for near in frared region. The photomultiplier tube detector can be switched to the InGaAs detector in the wavelength range from $700 \mathrm{~nm}$ to $1000 \mathrm{~nm}$ (the default switching wavelength is $870 \mathrm{~nm}$ ). The In GaAs detector can be switched to a $\mathrm{PbS}$ detector in the range from $1600 \mathrm{~nm}$ to $1800 \mathrm{~nm}$ (the default switching wave length is $1650 \mathrm{~nm}$ ). 


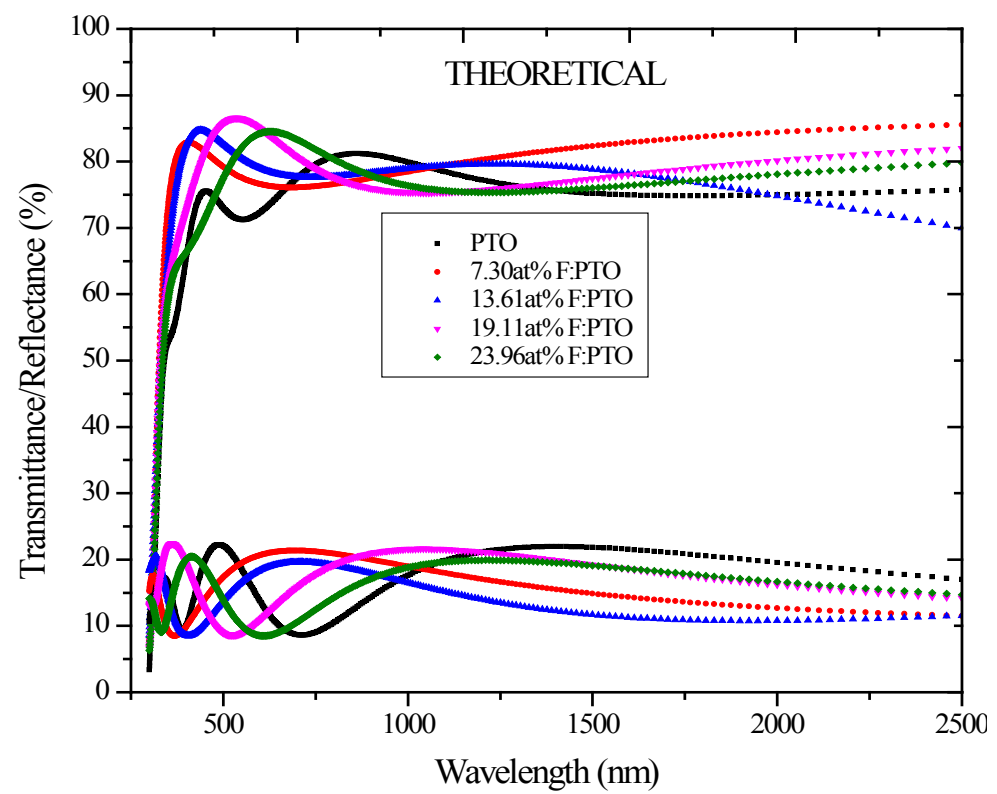

Figure 2(a). Experimental Spectral Transmittance and Reflectance of PTO film and F:PTO films of thickness $132.5 \pm 10 \mathrm{n} \mathrm{m}$ prepared at $450 \pm 10^{\circ} \mathrm{C}$

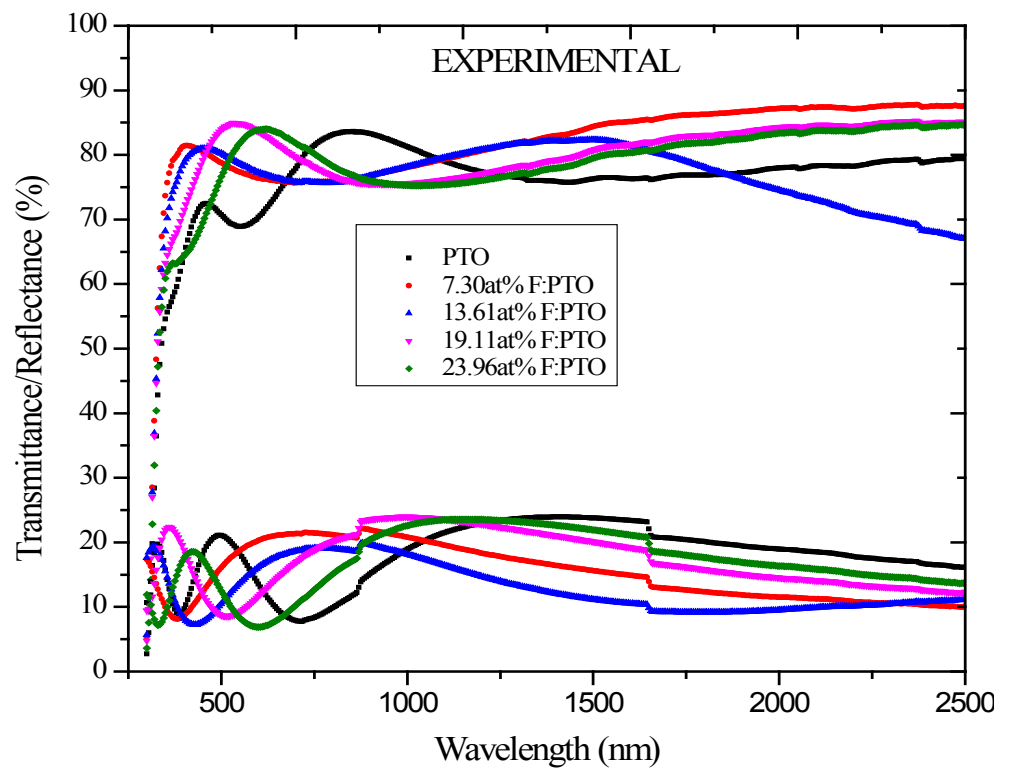

Figure 2(b). Computed SpectralTransmittance and Reflectance of PTO film and F:PTO films of thickness $132.5 \pm 10 \mathrm{~nm}$ prepared at $450 \pm 10^{\circ} \mathrm{C}$

The transparency at wavelengths of $355-495 \mathrm{~nm}$ had a maximum of $81.7 \%$ for $7.30 \mathrm{at} \%$ F:PTO which decreased with increasing $\mathrm{F}$ doping concentration to $64.2 \%$ for 23.96 at $\% \mathrm{~F}: \mathrm{PTO}$. This is due to the increase in fundamental absorption as photon striking increases with increase in carrier concentration[20]. The maximum transmittance observed in the 19.11 at\% F:PTO films may be attributed to a decrease in diffuse and multiple reflections caused by the increase in grain size and a reduction in light-scattering effect[21]. The film with the lowest resistivity had the lowest transmission, especially in the near infrared. A sharp fall in transmission at about $310 \mathrm{~nm}$ is due to the absorption of the glas s substrate[14].

The optical constants are reported in Figure 3 showing spectral refractive index $\mathrm{n}(\lambda)$ and extinction coefficient $\mathrm{k}(\lambda)$ for PTO film and F:PTO films. Dispersion analysis using a model for the dielectric susceptibility of the film consisting of Drude[22] and Kim terms[23] was used to model the measured reflectance and transmittance. The charge carriers set free by the donors or acceptors can be accelerated by very little energies and hence do respond to applied electric fields with frequencies in the infrared region[19]. Drude is a free electron contribution which describes the intraband contributions to the optical properties.

This model has two adjustable parameters: plasma frequency, $\Omega_{\mathrm{p}}$ and damping constant, $\gamma$. The plasma frequency is proportional to the square root of the carrier density and the damping constant is proportional to the inverse of the mobility. The Drude dielectric susceptibility $\chi_{\text {Drude }}$, expressed as a function of frequency $\omega$, is given as $[19,22]$ 


$$
\chi_{\text {Drude }}(\omega)=\frac{\Omega_{\mathrm{p}}{ }^{2}}{\omega^{2}+\mathrm{i} \omega \gamma}
$$

The one oscillator contribution developed by Kim contains four adjustable parameters: $\Omega_{\mathrm{TO}}$ resonance frequency, $\Omega_{\mathrm{p}}$ oscillator strength, $\Omega_{\tau}$ damping constant and Gauss-Lorentz-switch constant $\sigma$. $\sigma$ may vary between zero and infinity. For $\sigma=0$, a Gaussian line shape is achieved. A large value of $\sigma$ (larger than 5) leads to a Lorentzian line shape. The Kim oscillator models the weak broad interband absorption in the measured wavelength range. The interband dielectric susceptibility described by $\mathrm{Kim}$ is given by[20];

$$
\begin{gathered}
\chi_{\text {Kim, Oscillator }}=\frac{\Omega_{\mathrm{p}}{ }^{2}}{\Omega_{\mathrm{TO}}{ }^{2}-\omega^{2}-\mathrm{i} \omega \tau(\omega)} \\
\text { where } \tau(\omega)=\Omega_{\tau} \exp \left[-\frac{1}{1+\sigma^{2}}\left(\frac{\omega-\Omega_{\mathrm{TO}}}{\Omega_{\tau}}\right)^{2}\right]
\end{gathered}
$$

Model parameters were determined from the best fit between computed and experimental data using Scout software[19]. The best fit gives us directly the optical constants of the film under study. Figure 4 shows the experimental spectra and the fitted simulated spectra of 7.30at\% F:PTO. The thicknesses of the five samples obtained from fitting the experimental spectral data to theoretical spectral data based on dispersion analys is using the SCOUT software in the wavelength range $0.3-2.5 \mu \mathrm{m}$ was found to be $132.5 \pm 10 \mathrm{~nm}$. This was verified by the thicknesses obtained from SEM cross sections. The thicknesses obtained from the two different methods agree within a discrepancy of not more than $5 \%$.

In Figure 3, the refractive index of PTO was found to be around 1.98 at $500 \mathrm{~nm}$. It was also observed that the refractive index of all the films decreases with wavelength and then attains almost a constant value towards higher wavelengths [6].

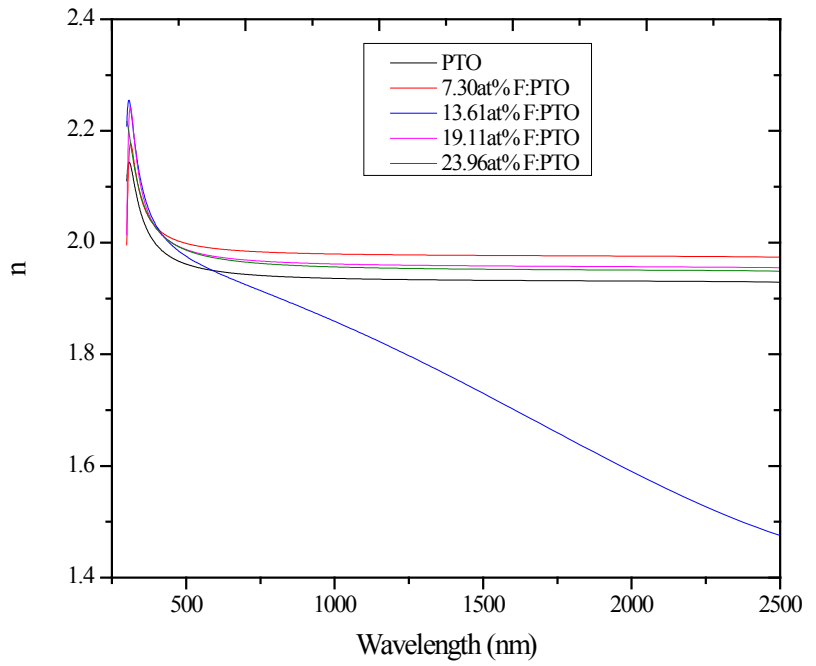

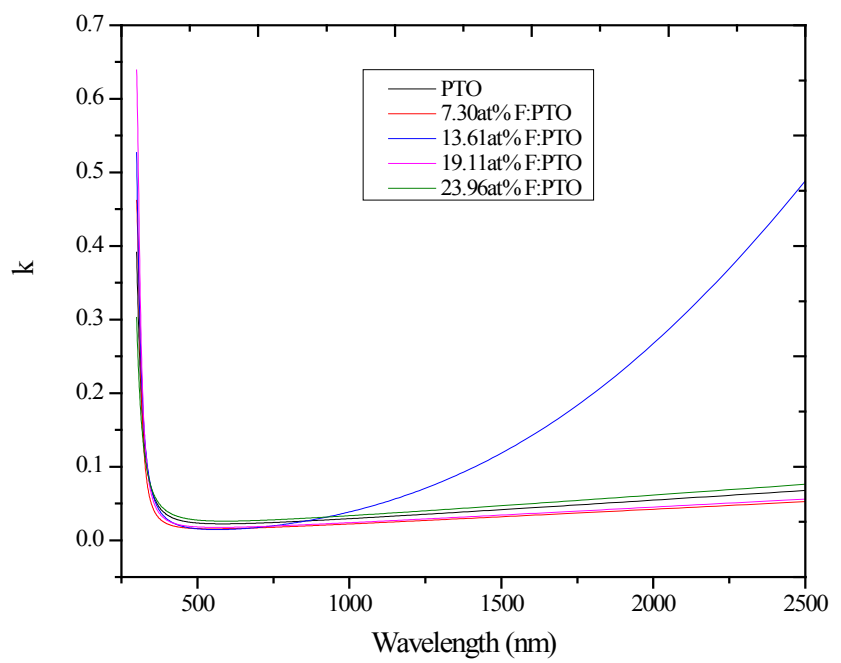

Figure 3. Spectral refractive index (n) and extinction coefficient $(\mathrm{k})$ of PTO film and F:PTO films prepared at $450 \pm 10^{\circ} \mathrm{C}$

For 13.61at\% F:PTO film, refractive index decreased further with increase in wavelength due to the increase in free charge carriers [19].

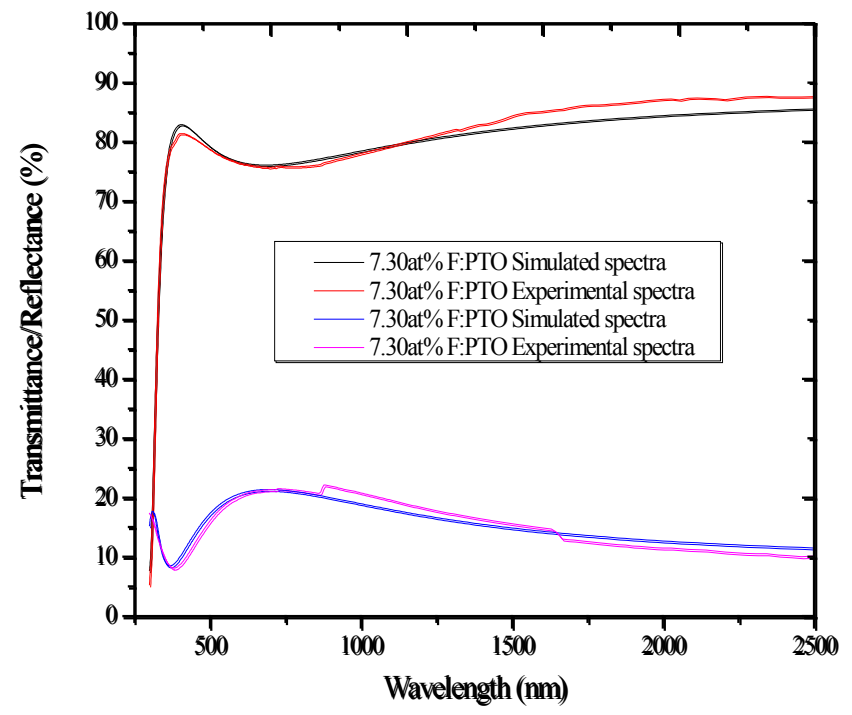

Figure 4. Best fit curve of Experimentaland Simulated spectra from Scout software for 7.30 at $\%$ F:PTO

Figure 5 shows spectral absorption coefficient $(\alpha)$ for PTO and F:PTO with four fluorine doping levels. For all the films, the absorption edge lies in the UV region and varies with the charge concentration. The samples show a slight decrease in the absorption coefficient for $\alpha<10^{4} \mathrm{~cm}^{-1}$ for $\lambda>1000 \mathrm{~nm}$ for all samples apart from 13.61at $\% \mathrm{~F}: \mathrm{PTO}$ film which tends to increase as shown in Figure 5(i). The dependence on $\alpha$ on $\mathrm{h} v$ region is shown in Figure 5(ii). It is clear that the value of $\alpha$ increases with increasing photon energy in the range 0.5 $\mathrm{eV}-1.3 \mathrm{eV}$ but decreases for 13.61at\% F:PTO film. This may be due to variation in fluorine concentration in the films.

The optical bandgap, $E_{g}$, was determined using the standard formula[22,23]:

$$
\alpha h v \alpha\left(h v-E_{g}\right)^{\mathrm{n}}
$$




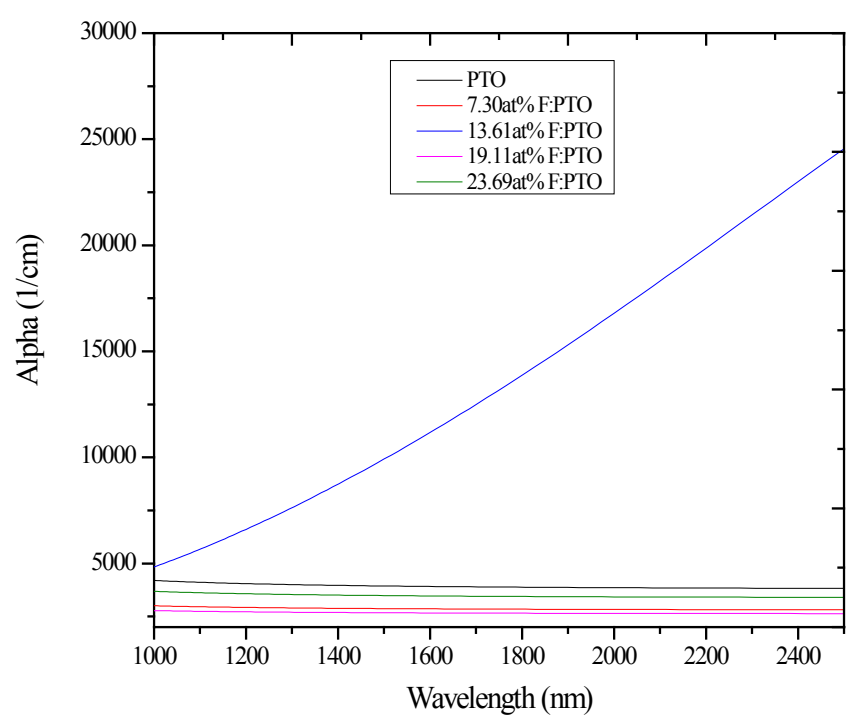

(i)

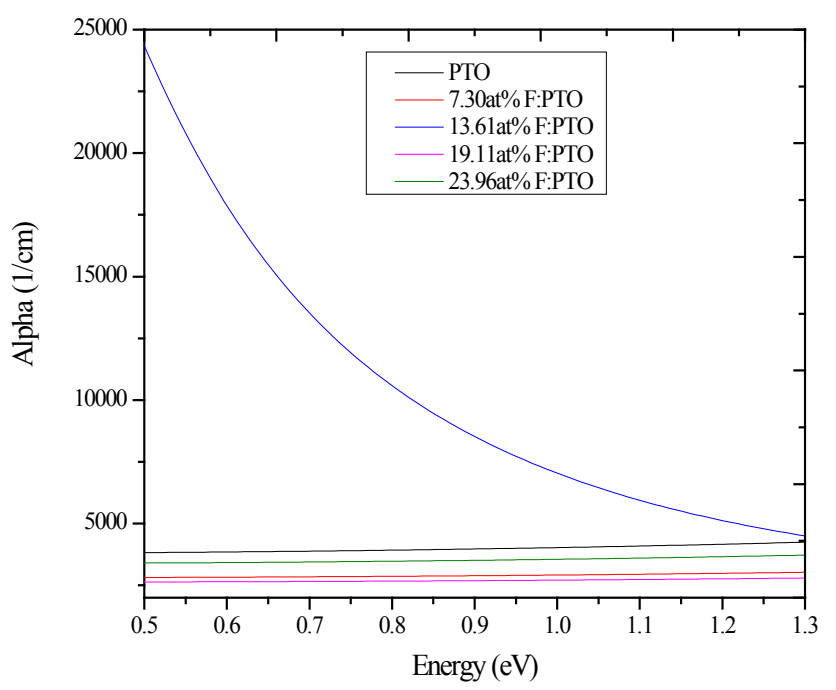

(ii)

Figure 5. Absorption coefficient $(\alpha)$ against wavelength (i) and Absorption coefficient ( $\alpha$ ) against Energy of PTO film and F:PTO films prepared at $450 \pm 10^{\circ} \mathrm{C}$

Figure 6 shows plots of $(\alpha h v) 2$ versus photo energy, hv, in the high absorption region. Extrapolation of the curve to hv $=$ 0 gave the direct band gap of PTO and F:PTO films in the range $3.945 \mathrm{eV}-4.014 \mathrm{eV}$. The bandgaps for 7.30at\% Where $\alpha=2 \pi k / \lambda \alpha=2 \pi k / \lambda$ is the absorption coefficient, $h v$, the photon energy, and $n=1 / 2$ accounts for the fact that the $\mathrm{d}$ irectly allowed transitions across the bandgap are expected to dominate

F:PTO and 13.61at\% F:PTO films are found to be wider than that of PTO, 19.11at\% F:PTO and 23.96at\% F:PTO. The increase in the energy gap is correlated with the Moss-Burstein effect and many body effects since the absorption edge of the films shifts to shorter wavelength[1]. It shows that ${ }_{E g}$, for the transparent thin film, increases with the carrier concentration. There is a good agreement with literature where the values are varying between 3.9 and $4.5 \mathrm{eV}$ depending on the dopants and preparation method[26-29].

This indicates an optimum level of Fluorine doping on the PTO film, causes a widening effect on the band gap and is attributed to be due to the gradual increase in carrier concentration and mobility[20,21].

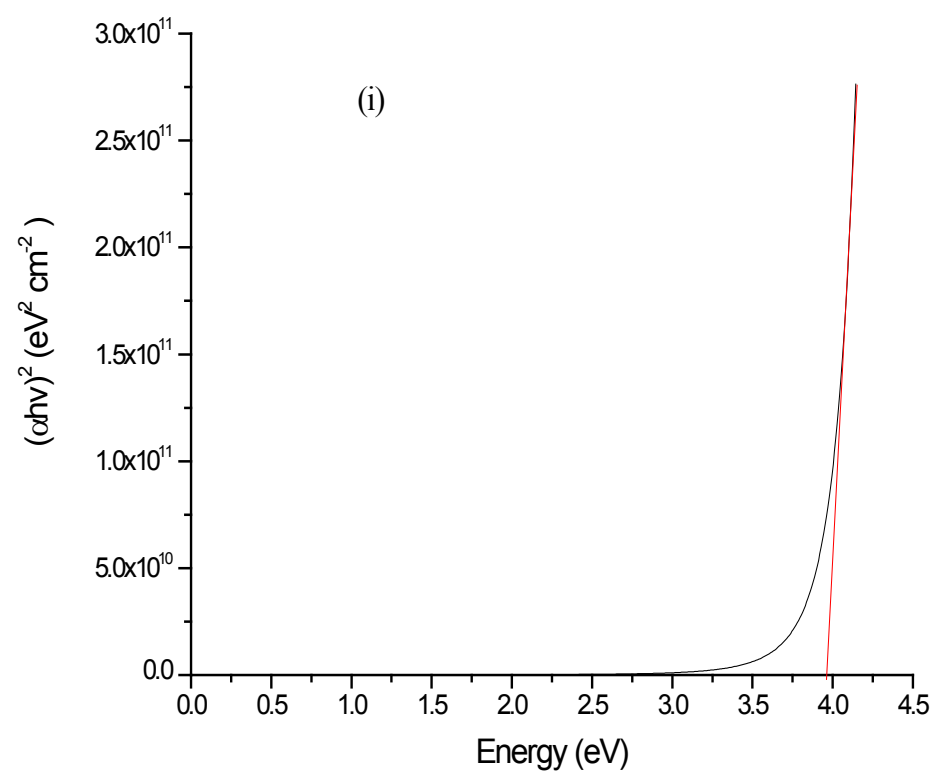



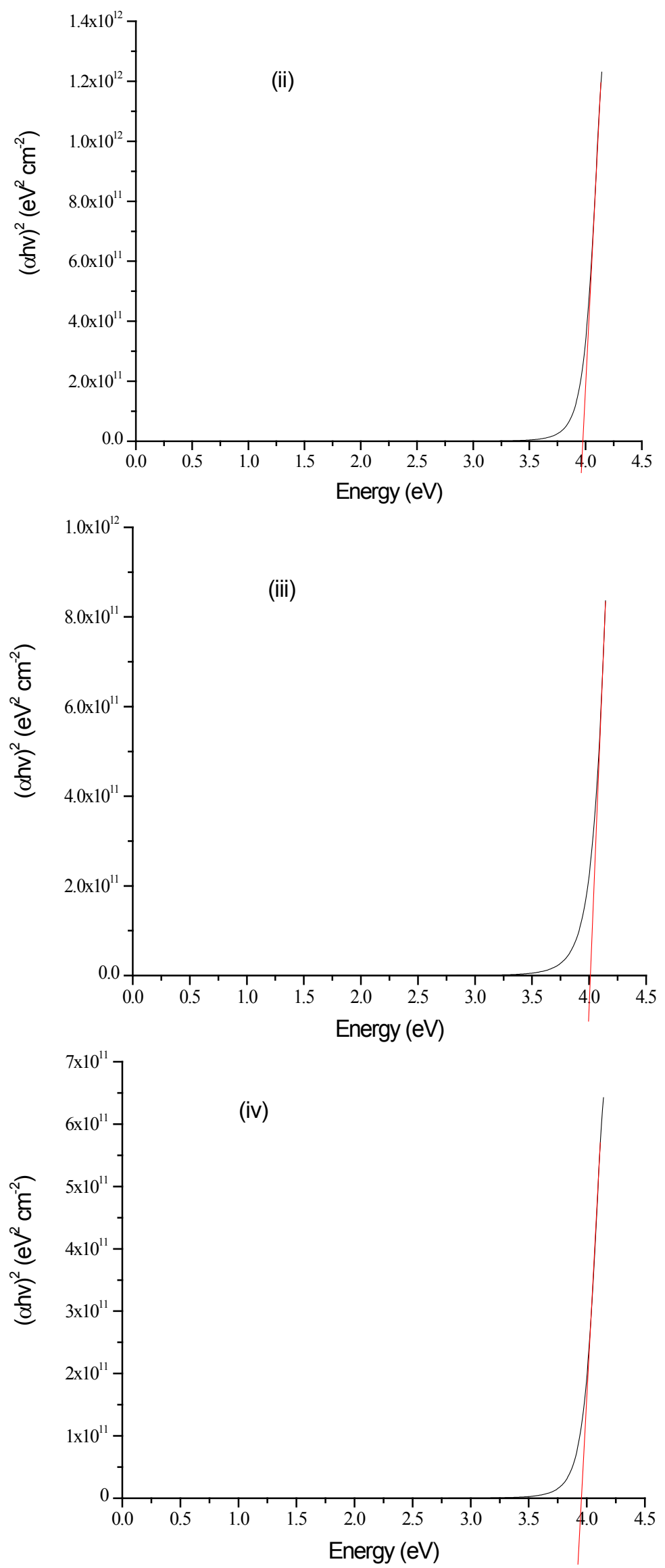


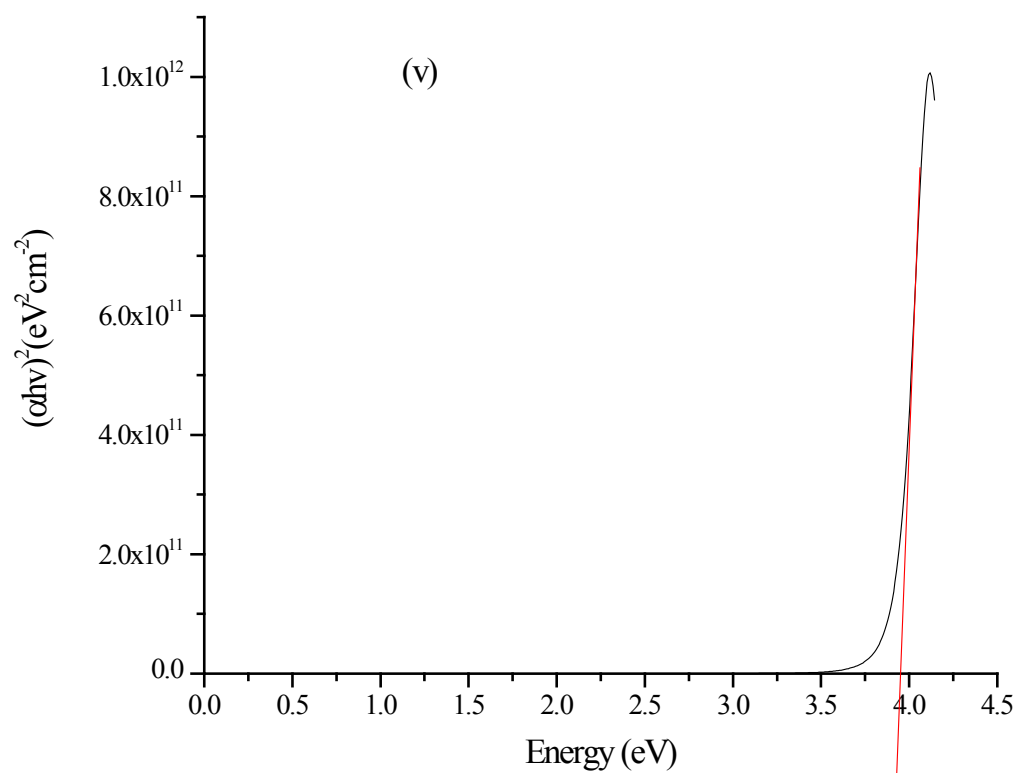

Figure 6. Energy bandgap of PTO and F:PTO films prepared at $450 \pm 10^{\circ} \mathrm{C}$, (i) PTO (ii) 7.30 at $\%$ F:PTO (iii) 13.61 at $\%$ F:PTO (iv) 19.1 lat $\%$ F:PTO (v) 23.96at \% F:PTO

Figure 7 shows the bandgap energy of PTO thin film and $F: P T O$ thin films with different atomic percentage of F. It is observed that the band gap energy increases with $F$ percentage up to 13.61 at\% F:PTO then it decreases with increase in the concentration of $\mathrm{F}$. This is in accordance with the findings of Mageto et.al, 2012 and Fatema et.al, 2011[17, 24]. This is due to the decrease in the number of charge carriers for higher $\mathrm{F}$ doping concentrations.

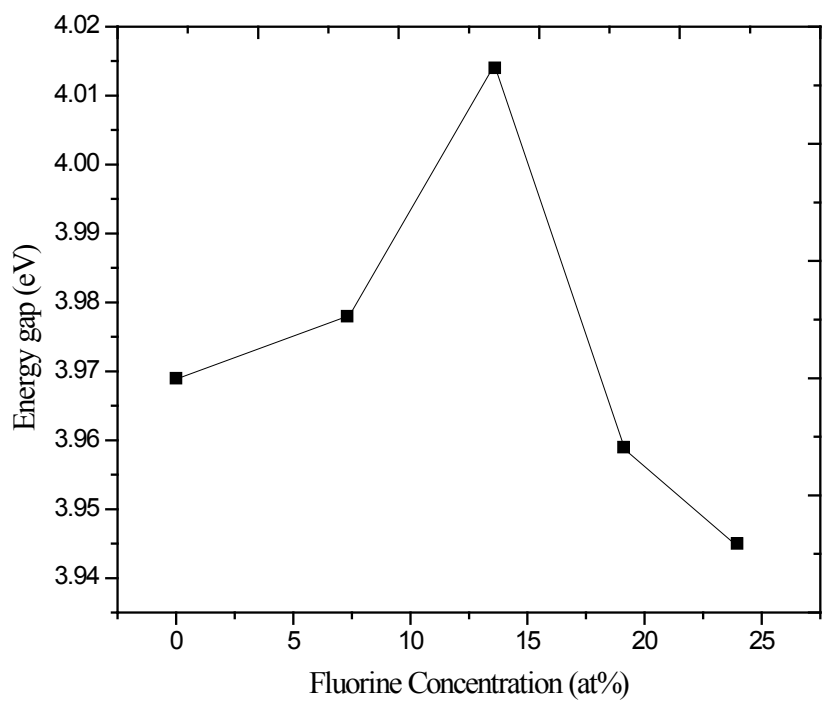

Figure 7. Energy gap versus F concentration

\subsection{Analysis of Electrical Properties}

Electrical properties were analy zed by use of Drude model basing on the results from the spectrophotometer.

The polarization $P$ induced by an externally applied electric field $E$, of the probing light wave in a homogeneous material is given by the electric susceptibility $\chi$;

$$
\mathrm{P}=\varepsilon_{0} \chi \mathrm{E}
$$

The dielectric function $\varepsilon$ connects the dielectric displacement and the electric field vector and is closely related to the susceptibility $\chi$;

$$
\mathrm{D}=\varepsilon_{0} \varepsilon \mathrm{E}
$$

where

$$
\varepsilon=1+\chi
$$

The frequency dependence of the susceptibility is very characteristic for a material since it incorporates vibrations of the electronic system and the atomic cores as well as contributions from free charge carriers. The doping of semiconductors leads to free charge carriers which can be investigated by IR spectroscopy. The response of the free carriers to oscillating electric fields can be described to a good approximation by the Drude model. The parameters of that model relate the concentration of the charge carriers and their mobility to properties of the dielectric function. After a model parameter fit of the simulated spectrum to measured data the carrier concentration and the mobility or resistivity can be computed[19]. The Drude model relates the macroscopic susceptibility to the microscopic quantities carrier concentration $n$ and mobility $\mu$;

$$
\begin{gathered}
n=\frac{4 \pi^{2} c_{0}^{2} \varepsilon_{0} m}{e^{2}} \Omega_{p}^{2}=3.35 \times 10^{14} \mathrm{~cm}^{-1} \Omega_{p}^{2} \\
\text { and } \mu=\frac{e}{m} \frac{1}{c_{0} \Omega_{\tau}}=1.96 \times 10^{3} \frac{1}{\Omega_{\tau}}
\end{gathered}
$$

where $e$ is the elementary charge $\left(1.6 \times 10^{-19} \mathrm{C}\right), \varepsilon_{0}$ is the permittivity of the free space $\left(8.854 \times 10^{-12} \mathrm{As} / \mathrm{Vm}\right)$ and $m$ the effective mass of the charge carriers $\left(m=0.3 m_{o}\right)[25]$ and $m_{0}$ is the mass of an electron. Resistivity can also be computed using the formula[16];

$$
\rho=\frac{\Omega_{\tau}}{2 \pi c_{0} \varepsilon_{0} \Omega_{P}^{2}}=6.0 \times 10^{1} \Omega \frac{\Omega_{\tau}}{\Omega_{P}^{2}}
$$

Figure 8 shows resistivity and carrier concentration as a function of the F content for PTO and F:PTO films with F content of $0-23.96 \mathrm{at} \%$. The distribution of resistivity is mainly related to that of carrier concentration. In particular, 
the resistivity markedly increased as the $\mathrm{F}$ content was increased from $0 \mathrm{at} \%$ and reached a maximum at a $\mathrm{F}$ content of $7.30 \mathrm{at} \%$. It then decreased rapidly as the $\mathrm{F}$ content was further increased, reaching a minimum at a $\mathrm{F}$ content of $13.61 \mathrm{at} \%$.

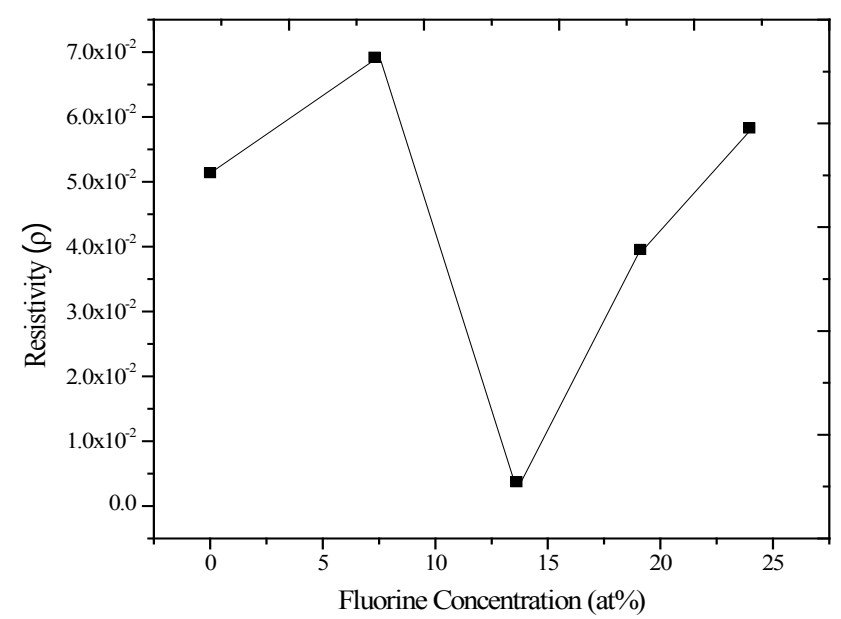

(i)

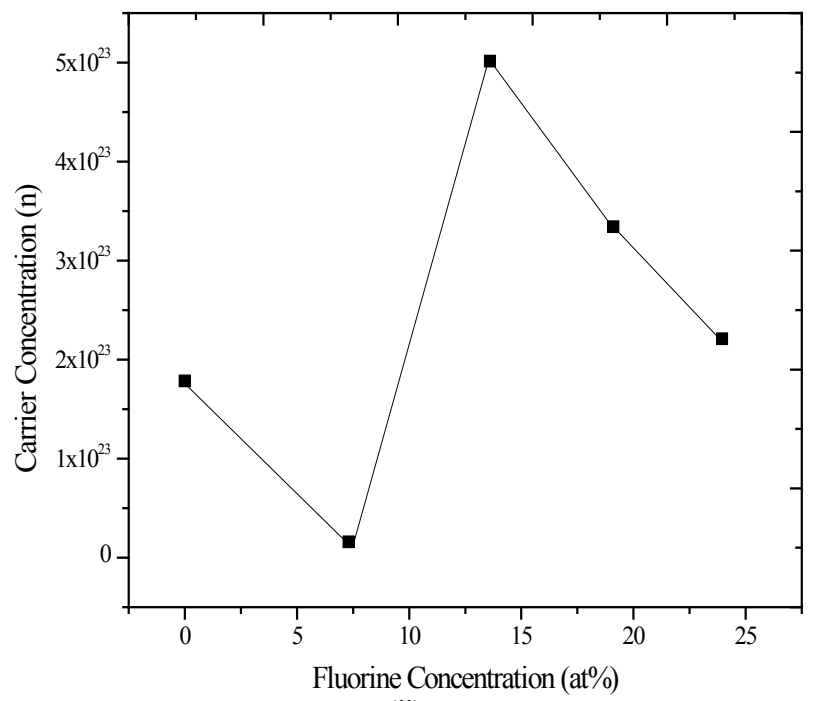

(ii)

Figure 8. (i) Resistivity versus F Concentration (ii) Carrier concentration versus F Concentration

The carrier concentration initially decreased as the $\mathrm{F}$ content was increased to $7.30 \mathrm{at} \%$. It is well known that a free electron acting as a carrier in $\mathrm{SnO}_{2}$ films is caused by two kinds of donors: a native defect such as an oxygen vacancy and $\mathrm{F} \mathrm{F}^{-}$ion on a substitutional site of an $\mathrm{O}^{2-}$ ion $[17$, 18]. Therefore, the drastic decrease of carrier concentration obtained in F:PTO films prepared with F content of 7.30 at $\%$ is possibly related to the following two origins: oxidation enhancement of films and carrier compensation due to doped Pd acting as an acceptor. The former may be caused by the spraying process being done in a fume chamber where oxygen is uncontrolled. Consequently, the decrease of carrier concentration may be caused by either extinction of oxygen vacancies or free electron trapping due to the adsorption of oxygen on the grain boundary and/or the surface of films. The latter, carrier compensation due to doped Pd acting as an acceptor, may be explained by the doping of $\mathrm{Pd}$ into $\mathrm{SnO}_{2}$ which may cause carrier compensation when a $\mathrm{Pd}^{2+}$ ion occupies the site of an $\mathrm{SnO}^{4+}$ ion. Although the decrease of carrier concentration with increases of $\mathrm{F}$ content up to $7.30 \mathrm{at} \%$ can be explained by a mechanis $\mathrm{m}$ where Pd acts as an acceptor, it may be difficult to apply this explanation for higher fluorine doping concentrations. In addition, if Pd acts as an acceptor, it is difficult to account for the increase of carrier concentration and its high value observed for films prepared with $\mathrm{F}$ contents above $7.30 \mathrm{at} \%$, as shown in Figure 7 . We suggest that this observed increased carrier concentration with a high value is related to the formation of ternary compounds, for example, $\mathrm{F}_{2} \mathrm{SnO}$ composed of $2 \mathrm{~F}$ and $\mathrm{SnO}[18]$ and $\mathrm{PdSnO}$ composed of $\mathrm{Pd}$ and $\mathrm{SnO}[12]$. It has been reported that $\mathrm{F}: \mathrm{SnO}_{2}$ films prepared by spray pyrolys is exhibit a high carrier concentration[20]. As described above, the drastic increase of resistivity may be explained by both oxygen enhancement and $\mathrm{Pd}$ acceptor mechanis ms.

\section{Conclusions}

Highly transparent and conductive F-co-doped PTO thin films $\left(1.4 \sim 2.67 \times 10^{2} \Omega^{-1} \mathrm{~cm}^{-1}\right)$ were prepared with a fluorine concentration of $0-23.96 \mathrm{at} \%$ by spray pyrolys is technique from $\mathrm{SnCl}_{4}$ precursor solution. The resistivity of the co-doped films initially increased from $5.14 \times 10^{-2} \Omega \mathrm{cm}$ with increase in fluorine ( $7.3 \mathrm{at} \% \mathrm{~F}: \mathrm{PTO})$ to attain a maximum value of $6.92 \times 10^{-2} \Omega \mathrm{cm}$ and drastically decreased to $3.74 \times 10^{-3} \Omega \mathrm{cm}$ for higher level doping (13.61at\% F:PTO) before gradually increasing again with increase in fluorine concentration. The resistivity achieved for the films doped with 13.61at\% F:PTO is the lowest for these films from $\mathrm{SnCl}_{4}$ precursor. Initial codoping of PTO with Fluorine leads to the widening of the bandgap (3.969-4.014 eV) which decreases on further increase of fluorine concentration to $3.945 \mathrm{eV}$. This is due to the increase in dopant concentration. The average transmittance of the F:PTO films in the visible region was $83 \%$. Increase in fluorine concentration also leads to decrease in transmittance in the visible wavelengths with high transmittance of the 19.1 lat $\% \mathrm{~F}: \mathrm{PTO}$ found to be $88 \%$.

\section{ACKNOWLEDGEMENTS}

The authors would like to thank the National Council of Science and Technology, Kenya, for their financial support towards carrying out this research. We also acknowledge the support from University of Nairobi technical team, Mr. Bonface Muthoka, Mr. Dickson Omucheni, Miss Everline Akinyi; and Miss Antonnette Omukaga, for their continuous ass istance when carrying out the experiments.

\section{REFERENCES}


[1] Tadatsugu M, Takashi Y, Yukinobu T, Toshihiro $M$. Transparent conducting zinc-co-doped ITO films prepared by magnetron sputtering. Thin Solid Films. 2000; 373:189-194

[2] Young SK, Ansari SG, AnsariZA, Rizwan W, Hyung-Shik S. A simple method to dep osit palladium doped $\mathrm{SnO}_{2}$ thin films using plasma enhanced chemical vapor deposition technique. American Institute of Physics, Rev. Sci. Instrum. 2010; 81: 113903

[3] R. Díaz. Tin oxide thin films: electronic properties and growth mechan ism under electrochemical control. University of Barcelona, Barcelona, Spain. (2002).

[4] Chitra A, Takwale MG, Bhide VG, Shailaja M, Kulkarni SK. Effect of $\mathrm{Sn}$ incorporation on the growth mechanism of sprayed $\mathrm{SnO}_{2}$ films. American Institute of Physics, J. Appl. Phys. 1991; 70: 7382

[5] Mishra RL, Sheo KM, Prakash SG. Optical and gas sensing characteristics of tin oxide nano-crystalline thin film. Journal of Ovonic Research. 2009; . 5:4: 77 - 85

[6] Shamala KS, Murthy LCS, Narasimha KR. Studies on tin oxide films prepared by electron beam evaporation and spray pyroly sis methods. Indian Academy of Sciences, Bull. Mater. Sci. $2004 ; 27: 3,295-301$

[7] Shadia JI, Riy ad NAB. Effect of Processing on the Electrical Properties of Spray-Deposited $\mathrm{SnO}_{2}:$ F Thin Films. American Journal of Applied Sciences. 2008; 5 (6): 672-677

[8] Jebbari N, Kamoun NT, Bennaceur R. Effect of SnCl4 concentration on $\mathrm{SnO}_{2}: \mathrm{F}$, deposited by chemical spray pyroly sis. International Renewable En ergy Congress, Sousse, Tunisia. 2010

[9] Agashe C, Marathe RB, Takwale GM, Bhide GV. Structural properties of $\mathrm{SnO}_{2}: \mathrm{F}$ films deposited by spray pyrolysis technique. Thin Solid Films. 1988; 164: 261-264

[10] Mohammad TM. Performance and characteristics of $\mathrm{Al}-\mathrm{PbS} / \mathrm{SnO}_{2}: \mathrm{F}$ selective coating system for photothermal energy conversion. Solar Energy Mater. 1990; 20:297-305

[11] Rakhshani EA, Makdisi Y, Ramazaniy an A.H. Electronic and optical properties of fluorine-doped tin oxide films. J. Applied Phys. 1998; 83: 1049-1057

[12] Boshta M, Mahmud FA, Sayed MH. Characterization of sprayed $\mathrm{SnO}_{2}$ : $\mathrm{Pd}$ thin films for gas sensing applications. Journal of Ovonic Research. 2010; 6 (2): 93 - 98

[13] Stanislav R, Ekaterina R, Tamara S. Electrophysical Properties of Gas Sensitive Films $\mathrm{SnO}_{2}$ Doped with Palladium, Sensors \& Transducers Magazine. 2004;.40 (2):145-151

[14] Sandipan R, Gupta PS, Gurdeep S. Electrical and Optical properties of sol-gel prepared Pd-doped $\mathrm{SnO}_{2}$ thin films: Effect of multiple layers and its use as room temperature methane gas sensor. Journal of Ovonic Research. 2010 February; 6(1): 63-74

[15] Joong-Ki C, In-Sung H, Sun-Jung K, Joon-Shik P, Soon-Sup $\mathrm{P}$, Uny ong Jeongc et.al. Design of selective gas sensors using electrospun Pd-doped $\mathrm{SnO}_{2}$ hollow nanofibers. Sensors and Actuators B. 2010; 150:191-199

[16] Wan ZS, Muhamad MS, Ashkan S, Mohd AY. Structural, Optical and Electrical Properties of Fluorine Doped Tin Oxide Thin Films Deposited Using Inkjet Printing Technique. Sains Malaysiana. 2011; 40(3): 251-257

[17] Mageto M, Mwamburi M. The influence of Al doping on optical, electrical and structural properties of transparent and conducting $\mathrm{SnO}_{2}$ : Al thin films prepared by spray pyrolysis technique. Elixir Chem. Phys. 2012; 53:11922-11927

[18] Elangovan E., Ramamurthi K. Optoelectronic properties of spray deposited $\mathrm{SnO}_{2}: \mathrm{F}$ thin films for window materials in solar cells. J. Optoelectronics and Advanced Materials. 2003; $5(1): 45-54$

[19] W. Theiss, in: W. Theiss (Ed.), Scout Thin Film Analysis Software Handbook, Hard and Software, Aachen, Germany, 2001 www.mtheiss.com, Pg. 54-57

[20] Yousaf SA, Ali S. The effect of fluorine doping on optoelectronic properties of tin-dioxide $\left(\mathrm{F}: \mathrm{SnO}_{2}\right)$ thin films. Coden Jnsmac. 2009; 48(1 \& 2), 43-50

[21] Sankara NS, Santhi B, Sundareswaran S, Venkatakrishnan KS. Studies on Spray Deposited $\mathrm{SnO}_{2}, \mathrm{Pd}: \mathrm{SnO}_{2}$ and F: $\mathrm{SnO}_{2}$ Thin Films for Gas Sensor Applications, Synthesis and Reactivity in Inorganic. Metal-Organic, and Nano-Metal Chemistry. 2006;36(1):131-135

[22] Ashcroft NW, Mermin ND. Solid State Physics. CBS Publishing, Philadelphia, USA. 1976; Pg. 18

[23] Kim CC, Garland JW, Abad H, Raccah PM. Modeling the optical dielectric function of semiconductors: extension of the critical-point parabolic-band approximation. Phys. Rev. B. 1992; $45: 11749$

[24] Fatema RC, Shamima C, Firoz H, Tahmina B. Optical properties of undoped and indium-doped tin oxide thin films. Journal of Bangladesh Academy of Sciences. 2011;35(1): 99-111

[25] Roy GG, Criteria for Choosing Transparent Conductors, mrs bulletin/au gust 2000, Available: http://www.mrs.org/publica tions/bulletin

[26] B. Stjerna, E. Olsson, C.G. Granqvist, J. Appl. Phys. 76 (1994) 3797.

[27] E. Shanthi, A. Banerjee, V. Dutta, K.L. Chopra, J. Appl. Phys. 53 (1982) 1615.

[28] E. Shanthi, V. Dutta, A. Banerjee, K.L. Chopra, J. Appl. Phys. 51 (1980) 6243.

[29] A. De, S. Ray, J. Phys. D: Appl. Phys. 24 (1991) 719.

[30] I. Saadeddin, H.S. Hilal, B. Pecquenard, J. Marcus, A. Mansouri, C.

[31] Labrugere, M.A. Subramanian, G. Campet, Solid State Sci. 8 (2006) 7. 